\title{
BMJ Open Retrospective cohort study of the association between maternal employment precarity and infant low birth weight in women in the USA
}

\author{
Divya Patil, ${ }^{1}$ Daniel A Enquobahrie, ${ }^{1}$ Trevor Peckham, ${ }^{2}$ Noah Seixas, ${ }^{2}$
} Anjum Hajat (1) ${ }^{1}$

To cite: Patil D,

Enquobahrie DA, Peckham T, et al. Retrospective cohort study of the association between maternal employment precarity and infant low birth weight in women in the USA. BMJ Open 2020;10:e029584. doi:10.1136/ bmjopen-2019-029584

- Prepublication history and additional material for this paper are available online. To view these files, please visit the journal online (http://dx.doi. org/10.1136/bmjopen-2019029584).

Received 01 February 2019 Revised 08 November 2019 Accepted 11 December 2019

Check for updates

(c) Author(s) (or their employer(s)) 2020. Re-use permitted under CC BY-NC. No commercial re-use. See rights and permissions. Published by BMJ.

${ }^{1}$ Department of Epidemiology, University of Washington School of Public Health, Seattle, Washington, USA

${ }^{2}$ Department of Environmental and Occupational Health

Sciences, University of

Washington, School of Public

Health, Seattle, Washington, USA

Correspondence to

Dr Anjum Hajat;

Anjumh@uw.edu

\section{ABSTRACT}

Objectives To investigate the association between maternal employment precarity and infant low birth weight (LBW), and to assess if this association differs by race/ ethnicity.

Methods Data were collected from 2871 women enrolled in the National Longitudinal Survey of Youth 1979 and the National Longitudinal Survey of Youth 1979 Children and Young Adult Cohort. Employment precarity was evaluated using a summary variable that combined several employment attributes: availability of employersponsored insurance, income, long shifts, non-daytime shifts, availability of employer sponsored training or educational benefits and membership in a union or collective bargaining unit. Employment precarity scores (a sum of the number of negative employment attributes) were categorised into low (0-2), medium (3) and high (46). LBW was defined as weight less than $2500 \mathrm{~g}$ at birth. Modified Poisson models were fit to calculate risk ratios and $95 \%$ Cls and adjusted for maternal age, race/ethnicity, educational attainment, nativity, prepregnancy body mass index, alcohol consumption, smoking during pregnancy and infant year of birth. We assessed effect modification by maternal race/ethnicity using a composite exposurerace variable.

Results Women with high employment precarity had higher risk of a LBW delivery compared with women with low employment precarity (RR: $1.48,95 \% \mathrm{Cl}: 1.11$ to 1.98 ). Compared to non-Hispanic/non-black women with low employment precarity, non-Hispanic black women (RR: 2.68; 95\% Cl: 1.72 to 4.15), Hispanic women (RR: 2.53; $95 \% \mathrm{Cl}: 1.54$ to 4.16 ) and non-Hispanic/non-black women (RR: $1.46 ; 95 \% \mathrm{Cl}: 0.98$ to 2.16 ) with high employment precarity had higher risk of LBW.

Conclusions We observed higher risk of LBW in pregnancies of women with high employment precarity; this association was stronger among black and Hispanic mothers compared to non-Hispanic/non-black women. Findings of this study can be used to inform antenatal care and identify workplace policies to better support women who work during pregnancy.

\section{INTRODUCTION}

Low birth weight (LBW) is associated with many chronic conditions in later life,
Strengths and limitations of this study

- Our study is the first to use a multidimensional definition of employment precarity to study its association with low birth weight.

- Data were collected from a diverse cohort of women living in the USA, allowing us to examine the racial/ ethnic differences in the association between employment precarity and low birth weight

- This study could benefit from data on more dimensions of employment precarity and additional confounding variables.

including obesity, hypertension and coronary heart diseases. ${ }^{12}$ Infants born with LBW are at a higher risk of death in the first year of life and lower educational attainment and income earnings in adult life. ${ }^{3-5}$ The prevalence of LBW deliveries in the USA has remained stagnant for more than two decades, with proportions recorded at about $7 \%$ in 1990 and $8 \%$ in $2016 .{ }^{6}$ LBW disparities have also been recorded between racial/ ethnic groups; in 2016, 13.7\% of infants born to non-Hispanic black women were LBW, compared to $7.0 \%$ and $7.3 \%$ of those born to non-Hispanic white and Hispanic mothers, respectively. ${ }^{6}$ While some exposures (such as income, maternal chronic conditions before and during pregnancy) and behavioural factors (including smoking) have been established as risk factors of LBW, the stable prevalence over time and observed disparities in LBW suggest that further examination of potential risk factors should be considered. ${ }^{7}$

As, of 2016, women made up $46.8 \%$ of the total US labour force; among women in their prime reproductive age (25-34 years), nearly $75 \%$ were working. A growing number of studies have investigated maternal occupational exposures during pregnancy and birth outcomes. ${ }^{89}$ Several studies have found 
associations with abnormal birth outcomes and workrelated chemical exposures (eg, solvents, toxic metals) and/or physical hazards (eg, lifting, standing for long durations), although contradictory evidence exists. ${ }^{10-14}$ A smaller number of studies have found associations between LBW and work-related psychosocial exposures, for example, job strain as measured by the job demand/ control model. ${ }^{15-17}$ These studies all focus on job content and working conditions, that is, the nature of tasks and the physical and social environment in which work takes place; however, the relational and contractual aspects of employment have received less attention as potential risk factors for LBW. This is despite widespread concerns that the nature and structure of employment have dramatically changed in recent decades towards more flexible, less secure employment arrangements. ${ }^{18-21}$ Indeed, a growing body of research has identified the terms and conditions of the employee-employer relationship-which determine contract type, wages and benefits, hours, schedule, mobility opportunities and workplace power dynamicsas important job-related determinants of a host of physical and mental health outcomes. ${ }^{22} 23$

Some indicators of employment conditions, when evaluated in isolation, have been associated with higher risk of LBW; this includes atypical or non-permanent contracts, long working hours and shift work. ${ }^{14}{ }^{24}$ However, jobs typically have several simultaneously occurring employment conditions, and it is the aggregation of these features that likely determine a worker's experience-such as whether a job is health-enhancing or deleterious. In other words, studies focused only on a single characteristic would fail to capture the complexity of contemporary employment arrangements. This has led to increased research attention to the multidimensional construct of precarious employment. ${ }^{22}$ Employment precarity can be defined as an accumulation of poor employment conditions that produce an experience of unstable, insecure employment, with inadequate wages and benefits and lacking social protections. ${ }^{25}{ }^{26}$ In this study we conceptualised precarity based on a framework used in a growing number of health studies in the European Union which includes the following seven dimensions: (1) employment stability, (2) material rewards, (3) workers' rights and social protections, (4) working time arrangements, (5) training and employability opportunities, (6) collective organisation and (7) interpersonal power relations. ${ }^{26} 27$

While employment precarity has been linked to several adverse health outcomes, no studies have applied this multidimensional construct to the study of LBW. ${ }^{22}$ Precarious employment can influence birth outcomes through several mechanisms. Most directly, precarious employment may cause maternal stress, due to job insecurity, lack of control or powerlessness. ${ }^{26}{ }^{28}$ Perinatal stress is thought to impact the hypothalamic pituitary adrenal axis and affect fetal growth during pregnancy. ${ }^{29-31}$ Furthermore, workers in precarious employment arrangements may have difficulty obtaining sufficient and stable income, as well as non-wage benefits. ${ }^{26}{ }^{32}$ This material deprivation could lead to impoverished living conditions or poor nutrition, which have been linked to LBW. ${ }^{33} 34$ Lastly, unpredictable work arrangements and the lack of paid leave may create barriers for women to receive timely prenatal care. ${ }^{35}$

The few studies evaluating employment characteristics (such as job strain and occupational sector) and birth outcomes were conducted outside of the USA, where different labour laws and social safety nets exist. ${ }^{3637}$ Further, few studies considered potential differences by racial/ethnic groups despite observed disparities in LBW. ${ }^{38}{ }^{39}$ This is especially relevant given evidence that precarious employment is socially distributed such that women, people of colour, immigrants, younger workers, lower-skilled and lower-educated workers are disproportionately represented. ${ }^{40} 41$

This study is the first to use a multidimensional definition of employment precarity to examine its association with infant LBW using a large, nationally representative cohort in the USA. We further examined differences in this association by race/ethnicity.

\section{METHODS}

The National Longitudinal Study of Youth 1979 (NLSY79), conducted by the US Bureau of Labor Statistics, is a nationally representative longitudinal cohort $(n=12686)$ of individuals born between 1957 and 1964, and living in the USA at the start of the survey in 1979. The NLSY79 Children cohort $(\mathrm{n}=11521)$ began in 1986 and surveyed all female respondents in the original cohort about their pregnancies and the health of their children; like the parent study (NLSY79), this survey was administered every year until 1996, after which it was administered every 2 years. The NLSY79 Children is linked to the original NLSY79 Cohort using the mother's ID to allow linkages across mother-infant pairs. At the start of data collection, $50 \%$ of the NLSY79 Cohort were females. As of 2014, the retention rate for the entire cohort is $71.0 \%$.

This study included women enrolled in the NLSY79 Cohort and consented to having their child included in the NLSY79 Children cohort. Women were excluded if they did not have a singleton first child born between 1978 and $2014(\mathrm{n}=1353)$, did not list at least one job in the interview cycle preceding the birth year of their first child during the survey period (1979-2014) $(\mathrm{n}=1982)$, had an infant with a birth weight of $4500 \mathrm{~g}$ or more $(\mathrm{n}=30)$ or had missing information on three or more precarity dimensions that were used to calculate the exposure $(n=47)$. The final sample included in the analysis was 2871 women (figure 1). We excluded unemployed women to improve exchangeability of exposed and unexposed groups. The last birth of a first-born child occurred in 2007 for women in the NLSY79, resulting in complete ascertainment of first births for this cohort. The Institutional Review Board at the University of Washington deemed this study to be exempt. Data used in this study are freely available on the NLSY website. 


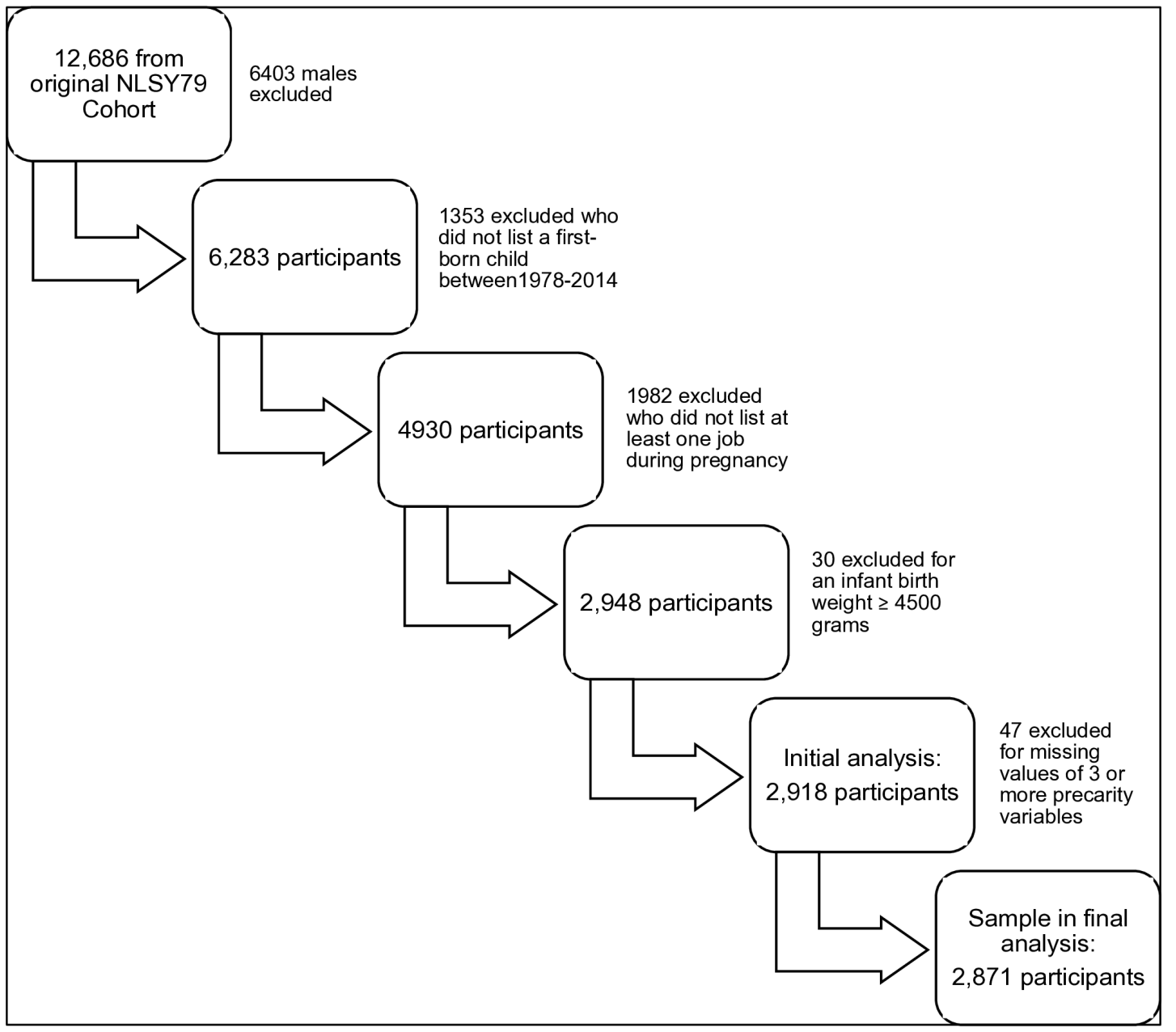

Figure 1 Exclusion criteria for participants in the National Longitudinal Study of Youth (NLSY79) cohort (1979-2007).

\section{Patient and public involvement}

Study participants were not involved in the development of the research question or the implementation of the study.

\section{Exposure}

The primary exposure was maternal employment precarity measured in the interview cycle preceding infant birth characterised by the following seven dimensions: (1) employment stability, (2) material rewards, (3) workers' rights and social protections, (4) working time arrangements, (5) training and employability opportunities, (6) collective organisation and (7) interpersonal power relations. ${ }^{26}{ }^{27}$ In this study, we operationalised employment precarity using available indicators of employment conditions within four of the seven dimensions. Material rewards was scored as high, medium or low based on two criteria: having employer-provided insurance (yes/no) and income in the last year adjusted for inflation to 1979 dollars (upper $50 \%$ of sample or lower $50 \%$ of sample). Those who had both employer-provided insurance and an income in the upper $50 \%$ of the sample were categorised as high (score of 0 ), those with either employer-provided insurance or an income in the upper
$50 \%$ of the sample were considered medium (1), and those with no employer-provided insurance or an income in the lower $50 \%$ of the sample were considered low (2). Working time arrangements was categorised as regular and irregular determined by participants' working hours per day ( $\leq 8$ hours, $>8$ hours), and time of day working (day time, non-day time). Participants working $\leq 8$ hours a day and during the day were considered to have regular working times $(0)$, those either working $>8$ hours or in a non-day time shift and those working both $>8$ hours and a non-day time shift were considered irregular (1-2). Training and employability opportunities were based on whether employers provided training or education to participants (0) or did not (1). Collective organisation was determined by an indicator of union membership (or whether wages are set by a union if union membership was not asked in that survey year), with membership scored as 0 and no membership as 1 . For women with more than one job, all characteristics pertain to the first job respondents reported. These four characteristics of employment are measured during the interview cycle prior to infant birth year and were summed to create an employment precarity composite measure ranging from 
0 to 6 , with higher scores reflecting greater employment precarity. Participants were further categorised into three groups based on their employment precarity composite score: low employment precarity (0-2), medium (3) and high (4-6). These cutoffs were determined using tertiles based on the distribution of the employment precarity scores. During some survey years, questions pertaining to these work precarity dimensions were not asked. For those years, data from the previous survey cycle was used in place of the missing information.

\section{Outcome}

The outcome of interest was maternal-reported infant LBW defined as birth weight of less than $2500 \mathrm{~g}$ (5 pounds, 8 ounces). An infant with birth weight of 2500-4500 g was considered normal birth weight.

\section{Covariates}

Several maternal covariates were included in the analyses: race/ethnicity (non-Hispanic/non-black, non-Hispanic black or Hispanic), age, educational attainment at time of pregnancy (less than high school, high school degree or more than high school), nativity (born in or outside of the USA), prepregnancy body mass index (BMI) calculated from self-reported height and weight, smoking during pregnancy (smoked at least once vs no smoking during pregnancy), alcohol consumption during pregnancy (any vs no alcohol consumption), as well as infant year of birth and infant sex (male or female). Data to break down the non-Hispanic/non-black group were not provided. Race/ethnicity was considered a confounder and an effect modifier and is being conceptualised as a proxy for racism and discrimination. All other covariates were included as confounders in the employment precarityLBW relationship. Maternal age and prepregnancy BMI were treated as continuous variables. Confounders and effect modifiers were chosen a priori based on existing literature on employment precarity and birth outcomes and based on a directed acyclic graph constructed for this analysis.

\section{Statistical analysis}

The NLSY79 provides sampling weights to adjust for oversampling, clustering and non-response. We used the weights to compute descriptive statistics (tables 1 and 2) but not estimates from multivariable models as there was a concern that parameter estimates would be biased. ${ }^{42} 43$ Descriptive statistics were used to compare sociodemographic and behavioural characteristics across employment precarity groups. For multivariable analysis we used modified Poisson regression models to estimate risk ratios and their corresponding 95\% CIs. These models were used because log binomial models did not converge in many instances and logistic models would overestimate parameter estimates since LBW is not rare. We evaluated precarious employment in three different ways. First, the association between each individual employment precarity component (material rewards, working time arrangements, employability opportunities and collective organisation), as well as variables used to create these components (income, insurance, shift time and hours worked), and LBW were examined. Second, we evaluated the composite precarity measure specified as a categorical variable (low, medium and high). Lastly, we evaluated a continuous specification of the composite measure.

We used three different adjustment models to delineate the different confounding effects of the sociodemographic and behavioural variables. Model 1 was an unadjusted generalised linear regression. Model 2 was adjusted for sociodemographic covariates including maternal age, maternal race/ethnicity, maternal educational attainment, maternal nativity and infant year of birth. Race/ethnicity, nativity and (in most cases) education were measured prior to employment and are likely strong confounders of the precarious employmentLBW association. Model 3 was adjusted for behavioural factors, including maternal prepregnancy BMI, maternal smoking, alcohol use during pregnancy and all the variables in model 2; this was considered our primary model.

It is possible that smoking and alcohol use are on the causal pathway between precarious employment and LBW, though it is unlikely that a woman would initiate smoking and higher levels of alcohol consumption during pregnancy. Smoking is often initiated in adolescents and early adulthood; one study indicated about $82 \%$ of smokers initiated smoking before age $21 .^{44}$ The mean age of our study participants was 24 years. As for alcohol consumption, it has been shown to stop or decrease dramatically after women learnt of their pregnancy. ${ }^{45}$ Using the three different adjustment models presented above allows readers to consider model 2 as primary if concerns about adjustment for smoking and alcohol persist.

We examined effect modification by race/ethnicity using the approach recommended by VanderWeele and $\mathrm{Knol}^{44}$ which created an employment precarity-race composite variable that combines the effect modifier and the exposure. The reference group was low employment precarity in non-Hispanic/non-black women. Risk ratios and corresponding 95\% CIs were calculated using fully adjusted modified Poisson models. As a sensitivity analysis, we collapsed the three-level employment precarity variable to two categories in order to make comparisons between low-precarity women and the medium-precarity and high-precarity women. Analyses were conducted using SAS V.9.4, and statistical significance was determined using the $\mathrm{p}<0.05$ cut-off.

\section{RESULTS}

Among study participants, 45\% ( $\mathrm{n}=1305)$ had low employment precarity, $31 \% \quad(\mathrm{n}=607)$ had medium employment precarity and 24\% (n=959) had high employment precarity. High and medium employment precarity had a higher prevalence of non-Hispanic black or Hispanic women compared to low employment precarity (table 1). High and medium employment precarity also had a 
Table 1 Weighted* sociodemographic characteristics of study participants from the NLSY79 cohort (1979-2007)

\begin{tabular}{|c|c|c|c|c|c|c|c|c|}
\hline & \multicolumn{2}{|c|}{ Entire sample $(n=2871)$} & \multicolumn{2}{|c|}{$\begin{array}{l}\text { Low employment precarity } \\
(n=1305)\end{array}$} & \multicolumn{2}{|c|}{$\begin{array}{l}\text { Medium employment } \\
\text { precarity }(n=607)\end{array}$} & \multicolumn{2}{|c|}{$\begin{array}{l}\text { High employment precarity } \\
(\mathrm{n}=959)\end{array}$} \\
\hline & $\mathrm{N}$ or mean & $\%$ or SE & $\mathrm{N}$ or mean & $\%$ or SE & $\mathrm{N}$ or mean & $\%$ or SE & $\mathrm{N}$ or mean & $\%$ or SE \\
\hline Age (years) & 24.3 & 0.78 & 26.4 & 0.89 & 23.5 & 1.48 & 21.5 & 0.81 \\
\hline Missing & 0 & 0 & 0 & 0 & 0 & 0 & 0 & 0 \\
\hline $\begin{array}{l}\text { Prepregnancy body } \\
\text { mass index }\left(\mathrm{kg} / \mathrm{m}^{2}\right) \dagger\end{array}$ & 23.2 & 0.45 & 230 & 0.28 & 23.7 & 0.65 & 22.0 & 1.20 \\
\hline \multicolumn{9}{|l|}{ Maternal race/ethnicity } \\
\hline Non-Hispanic black & 600 & 13.7 & 262 & 16.9 & 106 & 4.0 & 232 & 20.0 \\
\hline Hispanic & 495 & 6.3 & 212 & 5.9 & 95 & 12.3 & 188 & 4.9 \\
\hline $\begin{array}{l}\text { Non-Hispanic/non- } \\
\text { black }\end{array}$ & 1776 & 80.0 & 831 & 80.2 & 406 & 83.7 & 539 & 75.0 \\
\hline Outside of the USA & 189 & 5.3 & 83 & 2.2 & 36 & 11.8 & 70 & 3.0 \\
\hline Missing & 0 & 0 & 0 & 0 & 0 & 0 & 0 & 0 \\
\hline \multicolumn{9}{|c|}{ Maternal educational attainment } \\
\hline $\begin{array}{l}\text { Less than high } \\
\text { school }\end{array}$ & 479 & 13.1 & 144 & 3.7 & 78 & 27.3 & 257 & 12.7 \\
\hline High school & 1231 & 45.2 & 503 & 42.7 & 272 & 29.4 & 456 & 69.7 \\
\hline $\begin{array}{l}\text { More than high } \\
\text { school }\end{array}$ & 1161 & 41.7 & 658 & 53.6 & 257 & 43.3 & 246 & 17.7 \\
\hline Missing & 0 & 0 & 0 & 0 & 0 & 0 & 0 & 0 \\
\hline \multicolumn{9}{|l|}{ Infant sex } \\
\hline Missing & 144 & 4.1 & 75 & 7.0 & 24 & 1.1 & 45 & 2.5 \\
\hline \multicolumn{9}{|l|}{ Maternal alcohol } \\
\hline $\begin{array}{l}\text { Consumed at least } \\
\text { once }\end{array}$ & 1336 & 45.9 & 633 & 68.2 & 300 & 26.9 & 403 & 28.5 \\
\hline $\begin{array}{l}\text { Did not consume } \\
\text { alcohol }\end{array}$ & 1392 & 50.0 & 598 & 24.8 & 282 & 72.0 & 512 & 69.0 \\
\hline Missing & 143 & 4.1 & 74 & 7.0 & 25 & 1.2 & 44 & 2.5 \\
\hline
\end{tabular}

*Sample weights derived from the Bureau of Labour Statistics National Longitudinal Surveys custom weighting programme; means, SE and percentages are weighted, N's are unweighted.

†Body mass index calculated using self-reported height and self-report weight.

NLSY79, National Longitudinal Survey of Youth 1979.

higher proportion of less than high school educated women and smoking during pregnancy. High employment precarity had a smaller proportion of women who consumed alcohol compared to women with low employment precarity. Mean age at first birth was higher for low employment precarity (26.4, SE: 0.89 ) and lower for medium and high precarity (23.5, SE: 1.48 and 21.5, SE: $0.81)$. Overall, $85 \%(\mathrm{n}=2529)$ of participants delivered normal birth weight infants and $15 \%(\mathrm{n}=342)$ delivered
LBW infants (97 non-Hispanic black women, 67 Hispanic women and 178 non-Hispanic/non-black women delivered LBW infants).

Missingness for employment precarity variables was similar between outcome groups (table 2). All precarity variables had a unweighted missingness below $6 \%$ except for material rewards and insurance.

In the fully adjusted model 3, women reporting low and medium levels of material rewards had a higher risk 
Table 2 Weighted ${ }^{*}$ descriptive statistics of maternal employment precarity by per cent of low and normal birth weight, NLSY79 cohort (1979-2007)

\begin{tabular}{|c|c|c|c|c|c|c|c|c|}
\hline & & & \multicolumn{2}{|c|}{$\begin{array}{l}\text { Entire sample } \\
(\mathrm{n}=2871)\end{array}$} & \multicolumn{2}{|c|}{$\begin{array}{l}\text { Low birth weight } \\
(\mathrm{n}=342)\end{array}$} & \multicolumn{2}{|c|}{$\begin{array}{l}\text { Normal birth weight } \\
(n=2529)\end{array}$} \\
\hline & & & $\mathbf{N}$ & $\%$ & $\mathbf{N}$ & $\%$ & $\mathbf{N}$ & $\%$ \\
\hline \multirow{10}{*}{$\begin{array}{l}\text { Material } \\
\text { rewards }\end{array}$} & & High & 1102 & 44.0 & 129 & 79.6 & 973 & 37.7 \\
\hline & & Medium & 546 & 21.1 & 69 & 8.2 & 477 & 23.4 \\
\hline & & Low & 891 & 30.1 & 105 & 9.0 & 786 & 34.0 \\
\hline & & Missing & 332 & 4.7 & 39 & 3.2 & 293 & 5.0 \\
\hline & Income† & Upper 50\% & 1354 & 60.5 & 159 & 83.3 & 1194 & 41.2 \\
\hline & & Lower $50 \%$ & 1358 & 37.2 & 164 & 14.9 & 1195 & 56.4 \\
\hline & & Missing & 159 & 2.3 & 19 & 1.8 & 140 & 2.4 \\
\hline & Insurance & Yes & 1433 & 49.3 & 168 & 84.0 & 1265 & 43.1 \\
\hline & & No & 1258 & 48.3 & 151 & 14.3 & 1107 & 54.3 \\
\hline & & Missing & 180 & 2.5 & 23 & 1.7 & 157 & 2.6 \\
\hline \multirow{9}{*}{$\begin{array}{l}\text { Working time } \\
\text { arrangements } \ddagger\end{array}$} & & Regular & 1947 & 73.0 & 214 & 86.7 & 1733 & 70.5 \\
\hline & & Irregular & 796 & 24.4 & 114 & 12.6 & 682 & 26.5 \\
\hline & & Missing & 128 & 2.7 & 14 & 0.7 & 114 & 3.0 \\
\hline & Shift & Day shift & 2185 & 79.8 & 256 & 91.7 & 1929 & 77.6 \\
\hline & & Not day shift & 558 & 17.6 & 72 & 7.6 & 486 & 19.4 \\
\hline & & Missing & 128 & 2.7 & 14 & 0.7 & 114 & 3.0 \\
\hline & Hours per & $\leq 8$ & 2560 & 84.7 & 292 & 94.1 & 2268 & 83.0 \\
\hline & & $>8$ & 311 & 15.3 & 50 & 5.9 & 261 & 17.0 \\
\hline & & Missing & 0 & 0 & 0 & 0 & 0 & 0 \\
\hline \multirow{3}{*}{$\begin{array}{l}\text { Employability } \\
\text { opportunities§ }\end{array}$} & & Yes & 252 & 19.3 & 35 & 4.8 & 217 & 21.9 \\
\hline & & No & 2491 & 78.4 & 284 & 92.5 & 2207 & 75.9 \\
\hline & & Missing & 128 & 2.3 & 23 & 2.7 & 105 & 2.2 \\
\hline \multirow{3}{*}{$\begin{array}{l}\text { Collective } \\
\text { organisation }\end{array}$} & & Yes & 220 & 3.7 & 31 & 3.3 & 189 & 3.7 \\
\hline & & No & 2496 & 93.7 & 297 & 95.1 & 2199 & 93.4 \\
\hline & & Missing & 155 & 2.6 & 14 & 1.6 & 141 & 0.6 \\
\hline \multirow{5}{*}{$\begin{array}{l}\text { Employment } \\
\text { precarity }{ }^{* \star}\end{array}$} & & Low & 1305 & 45.0 & 152 & 80.9 & 1153 & 38.6 \\
\hline & & Medium & 607 & 30.5 & 75 & 8.6 & 532 & 34.5 \\
\hline & & High & 959 & 24.4 & 115 & 10.6 & 844 & 26.9 \\
\hline & & Continuous & 2871 & - & 342 & - & 2529 & - \\
\hline & & Missing & 0 & 0 & 0 & 0 & 0 & 0 \\
\hline
\end{tabular}

*Sample weights derived from the Bureau of Labor Statistics National Longitudinal Surveys custom weighting programme; means, SE and percentages are weighted, N's are unweighted.

tIncome was adjusted for inflation to the 1979 dollar value.

$\ddagger$ Women with regular working times had a day shift and worked $\leq 8$ hours per day. Women with irregular working times had a non-day shift or worked more than 8 hours per day or had both.

$\S$ Employability opportunities was defined as having employer-provided trainings or education.

ๆCollective organisation was defined as union membership.

${ }^{\star \star}$ Employment precarity was categorised based on scores from an index of individual employment characteristics; low: 0-2, medium: 3, high: 4-6.

NLSY79, National Longitudinal Survey of Youth 1979.

of LBW compared to those with a high level of material rewards (RR: 1.39 , 95\% CI: 1.01 to 1.91 and RR: $1.40,95 \%$ CI: 1.01 to 1.94 , respectively). Similarly, women with irregular working time arrangements had 1.27 (95\% CI: 1.00 to 1.61) times the risk of having a LBW infant compared to women with regular working times after adjustment.
Individual precarity variables were not associated with increased risk of LBW in this study, except for lack of health insurance in both adjusted models, and income in the lower $50 \%$ in model 2 (table 3 ).

The categorical precarity measure was positively associated with LBW after adjusting for confounders. Women 
Table 3 RRs and 95\% Cls for the association between employment precarity and low birth weight in a sample from the NLSY79 cohort (1979-2007)

\begin{tabular}{|c|c|c|c|c|c|c|c|c|}
\hline & & & \multicolumn{2}{|c|}{ Model 1* } & \multicolumn{2}{|c|}{ Model 2† } & \multicolumn{2}{|c|}{ Model 3‡ } \\
\hline & & & $\mathbf{R R}$ & $95 \% \mathrm{Cl}$ & $\mathbf{R R}$ & $95 \% \mathrm{Cl}$ & $\mathbf{R R}$ & $95 \% \mathrm{Cl}$ \\
\hline \multirow{5}{*}{$\begin{array}{l}\text { Material } \\
\text { rewards }\end{array}$} & & High & Ref & & Ref & & Ref & \\
\hline & & Low & 1.01 & 0.79 to 1.28 & 1.45 & 1.08 to 1.95 & 1.40 & 1.01 to 1.94 \\
\hline & Income & Upper 50\% & Ref & & Ref & & Ref & \\
\hline & Insurance & Yes & Ref & & Ref & & Ref & \\
\hline & & No & 1.02 & 0.83 to 1.26 & 1.29 & 1.02 to 1.64 & 1.30 & 1.00 to 1.69 \\
\hline \multirow{4}{*}{$\begin{array}{l}\text { Working time } \\
\text { arrangement§ }\end{array}$} & & Regular & Ref & & Ref & & Ref & \\
\hline & & Irregular & 1.30 & 1.05 to 1.61 & 1.22 & 0.99 to 1.50 & 1.27 & 1.00 to 1.61 \\
\hline & Shift & Day Shift & Ref & & Ref & & Ref & \\
\hline & & $>8$ & 1.41 & 1.07 to 1.86 & 1.23 & 0.93 to 1.61 & 1.32 & 0.97 to 1.81 \\
\hline \multirow{2}{*}{$\begin{array}{l}\text { Employability } \\
\text { opportunities }\end{array}$} & & Yes & Ref & & Ref & & Ref & \\
\hline & & No & 0.82 & 0.59 to 1.14 & 0.88 & 0.65 to 1.19 & 0.85 & 0.59 to 1.21 \\
\hline \multirow{2}{*}{$\begin{array}{l}\text { Collective } \\
\text { organisation }^{\star \star}\end{array}$} & & Yes & Ref & & Ref & & Ref & \\
\hline & & No & 0.84 & 0.60 to 1.19 & 0.88 & 0.63 to 1.24 & 0.89 & 0.59 to 1.34 \\
\hline \multirow{3}{*}{$\begin{array}{l}\text { Employment } \\
\text { precarity†† }\end{array}$} & & Low & Ref & & Ref & & Ref & \\
\hline & & Medium & 1.06 & 0.82 to 1.37 & 1.24 & 0.96 to 1.60 & 1.36 & 1.01 to 1.82 \\
\hline & & High & 1.03 & 0.82 to 1.29 & 1.32 & 1.03 to 1.70 & 1.48 & 1.11 to 1.98 \\
\hline
\end{tabular}

*Unadjusted generalised linear model of employment precarity and low birth weight.

†Model adjusted for maternal age, maternal race/ethnicity, maternal educational attainment, maternal nativity and infant year of birth.

$\ddagger$ Model adjusted for all covariates in model 2 plus maternal prepregnancy BMI, maternal smoking during pregnancy and maternal alcohol consumption during pregnancy.

$\S$ Women with regular working times had a day shift and worked $\leq 8$ hours per day. Women with irregular working times had a non-day shift or worked more than 8 hours per day or had both.

ПEmployability opportunities was defined as having employer provided trainings or education.

${ }^{* *}$ Collective organisation was defined as union membership.

††Employment precarity was categorised based on scores from an index of individual employment characteristics; low: 0-2, medium: 3, high: 4-6.

BMI, body mass index; NLSY79, National Longitudinal Survey of Youth 1979; RR, risk ratio.

with high and medium employment precarity were more likely to have a LBW infant compared to low employment precarity, after adjustment for all covariates (RR: 1.48, 95\% CI: 1.11 to 1.98 and 1.36, 95\% CI: 1.01 to 1.82 , respectively). The continuous measure of precarity showed a 1.17 (95\% CI: 1.05 to 1.30) times higher risk for LBW for a one point higher value in precarity after adjustment for all covariates.

We evaluated effect modification of the employment precarity-LBW association by race. The magnitude of the parameter estimates suggest that non-Hispanic black women had elevated LBW risk across all employment precarity levels compared to non-Hispanic/non-black women with low employment precarity (non-Hispanic black low precarity: $1.66,95 \%$ CI: 1.08 to 2.57 ; medium precarity: $3.04,95 \%$ CI: 1.87 to 4.95 ; high precarity: 2.68 ,
95\% CI: 1.72 to 4.15) (table 4). Hispanic mothers also had higher risk compared to non-Hispanic/non-black women with low precarity (Hispanic low precarity: 1.79, 95\% CI: 1.13 to 2.84 ; high precarity: 2.53 , 95\% CI: 1.54 to 4.16). The online supplementary table provides estimates comparing low precarity to the combined medium and high precarity group. These results are similar in magnitude to table 4 , but have improved precision.

\section{DISCUSSION}

In this study, we found that women with high employment precarity in the wave prior to or during pregnancy had a higher risk of giving birth to an LBW infant than women with low employment precarity. Of the individual employment characteristics examined, low material rewards and 
Table 4 RRs and 95\% Cls for the association between the composite employment precarity variable and low birth weight by maternal race/ethnicity in the NLSY79 cohort (1979-2007)*

\begin{tabular}{|c|c|c|c|c|c|c|c|c|c|}
\hline \multirow{2}{*}{$\begin{array}{l}\text { Employment } \\
\text { precarity }\end{array}$} & \multirow{2}{*}{$\begin{array}{l}\text { Non-Hispanic/ } \\
\text { non-black }\end{array}$} & \multirow[b]{2}{*}{$95 \% \mathrm{Cl}$} & \multirow[b]{2}{*}{$\mathbf{N}$} & \multirow{2}{*}{$\begin{array}{l}\text { Hispanic } \\
\text { RR } \\
\end{array}$} & \multirow[b]{2}{*}{$95 \% \mathrm{Cl}$} & \multirow[b]{2}{*}{$\mathbf{N}$} & \multirow{2}{*}{$\begin{array}{l}\text { Non- } \\
\text { Hispanic } \\
\text { black } \\
\text { RR }\end{array}$} & \multirow[b]{2}{*}{$95 \% \mathrm{Cl}$} & \multirow[b]{2}{*}{$\mathbf{N}$} \\
\hline & & & & & & & & & \\
\hline Low precarity & Ref & & 831 & 1.79 & 1.13 to 2.84 & 212 & 1.66 & 1.08 to 2.57 & 262 \\
\hline $\begin{array}{l}\text { Medium } \\
\text { precarity }\end{array}$ & 1.34 & $0.91-1.99$ & 406 & 1.65 & 0.87 to 3.12 & 95 & 3.04 & 1.87 to 4.95 & 106 \\
\hline
\end{tabular}

*Models are adjusted for maternal age, infant year of birth, maternal educational attainment during pregnancy, maternal nativity, maternal prepregnancy BMI, maternal smoking during pregnancy and maternal alcohol consumption during pregnancy.

NLSY79, National Longitudinal Survey of Youth 1979; RR, risk ratio.

irregular working time arrangements were associated with LBW. This elevated risk of LBW may be the result of employment-related stress or lack of access to resources due to these specific employment characteristics.

In addition, we found effect modification by maternal race/ethnicity, with elevated LBW risk in all employment precarity groups in non-Hispanic black women and the low and high employment precarity groups in Hispanic women when compared with non-Hispanic/non-black women with low precarity. Though the medium precarity Hispanic group did not have significant findings, this may be the result of a lack of statistical power. This suggests that health inequities exist between these subpopulations and could be a result of different stressors from employment precarity or a combination of workplace and additional life stressors, such as racism and discrimination that women of colour disproportionately experience. Furthermore, our results suggest that employment precarity may be one mechanism by which race/ethnic disparities in LBW occur. Labour market stratification results in more women and people of colour being employed in precarious employment ${ }^{41}$; thus, suggesting the importance of employment precarity as a mechanism by which health disparities are created and maintained. We are unable to test this hypothesis in our study; however, work in progress by our research team will examine this question in depth.

Our study is the first to investigate the association between a multidimensional maternal employment precarity construct and infant LBW. Findings were similar to studies examining traditional job stressors, such as job demand/control, that showed higher risk for adverse birth outcomes, such as LBW and preterm birth, in those with poorer quality jobs. ${ }^{1524} 36$ Some older studies and studies conducted outside of the USA did not find job stressors increased the risk for preterm birth and small for gestational age, suggesting that national and temporal contexts may influence the work-pregnancy outcome association. ${ }^{45} 46$ This study adds to a growing body of literature that aims to characterise multiple facets of employment quality which extends beyond the more traditional models of job stress, which focus on the psychosocial work environment. ${ }^{23} 47$ This study also adds to the literature on inequities in birth outcomes between racial/ethnic groups in the USA and emphasises the choice of reference groups when conducting research in regard to inequities. ${ }^{26} 283334$

Our study has several limitations. First, there is incomplete ascertainment of precarity as laid out by our conceptual framework. ${ }^{26} 27$ In particular, data within the NLSY for the dimensions of employment stability, interpersonal power relations and workers' rights and social protections is lacking; this is a part of a broader issue that few datasets have robust information on employment precarity. It is also possible that the existing variables we use to define the dimensions of precarity may not fully capture those dimensions. Additional research on defining precarity, specifically in the US context, is needed to better evaluate the measurement of this multidimensional construct. Second, misclassification of the exposure may have resulted from our approach to missing data, in which we used data from the year prior to populate missing data for a subsequent year. In a related concern, we explored multiple imputation methods for the material rewards dimension, but decided against it as both the amount of missing data (around 10\% unweighted) and the likely pattern of missing data (not missing at random) cannot be accommodated by most statistical software packages ${ }^{48}$ Regardless, some bias may result from missing data. Further, due to a lack of information on exact date of birth, precarity measures were taken in the interview cycle preceding the year of birth, thus may represent employment occurring as little as a few months before the child's birth to as much as 3 years prior to the birth. Therefore, the exposure may represent different clinically relevant time points for each participant. Also related to exposure misclassification, we do not believe that validity of self-reported employment characteristics would differ between outcome groups; thus resulting in non-differential misclassification. Although on average non-differential misclassification attenuates estimates towards the null, this may not be the case in our study given the other potential sources of bias. ${ }^{49} 50$ Another source of potential misclassification lies in the self-reported outcome data; however, a past study found high correlation between maternal recall of infant birth 
weight many years after pregnancy and birth weight obtained during pregnancy. ${ }^{51}$ Though some infants may be LBW as a result of a preterm birth, we did not have information on gestational age and could not distinguish between full term LBW and preterm LBW infants which may be important as these two birth outcomes may have different etiological pathways. Residual and unmeasured confounding was also a limitation. Data on pregnancy risk factors, partner's employment status, their fringe benefits and social support networks may also have been important unmeasured confounders. Sample sizes for racial and ethnic groups were small when assessing differences in the employment precarity-LBW association by subgroup, thus impacting our power to detect an effect. The NLSY uses a stratified random sampling approach, where participants are recruited only from certain areas of the nation. This clustering can bias our results, as people living in certain neighbourhoods may be more similar to each other than those living in different areas. In essence, this is a violation of the independence assumption applicable to most regression modelling approaches. Given our study population, the only approach to adjusting for clustering would be to gain access to the restricted geocoded data. We were unable to do this, thus we recognise that clustering may bias our results. The direction of bias, however, is predictable. Accounting for clustering will inflate the SEs, but should have minimal effect on the point estimates. Thus, the true CIs will likely be wider than what is presented in tables 3 and 4 which in some cases may result in non-statistically significant estimates. Thus we urge readers to view our regression results with healthy scepticism. Finally, sampling for this study was based on the population residing in the USA in 1979 and was employed during or prior to pregnancy. It may not be representative to today's sociodemographic distribution, or to the risk of LBW for those without a job or searching for employment.

Future research should focus on elucidating the employment precarity-LBW relationship through a more complete ascertainment of the exposure, including indicators from all seven precarity dimensions and a better assessment of the most relevant maternal employment period (eg, prior to or concurrent with pregnancy) for birth outcomes. Researchers interested in employment precarity and birth outcomes may also consider including data on things such as availability of paid family leave as these types of policies are of particular relevance to women as well as oversampling racial/ethnic groups to assess differences in subpopulations. Investigating the role of paternal employment precarity as an exposure for adverse birth outcomes may also be a fruitful avenue of research. Because the data we used did not distinguish between unemployed individuals looking for paid work and those not seeking employment, we could not include a reference group of women seeking employment. However, future research should consider including these women as a reference group, especially in an effort to further understand the gig economy.
Our findings support greater examination of employment precarity and health, especially as a larger proportion of women enter and remain in the workforce. The findings from studies of employment precarity could better inform prenatal care for working women. Clinicians treating women who plan to work during their pregnancy should consider asking their patients about their employment conditions and recommend resources that may mitigate the effects of employment precarity. Potential workplace policies or practices, such as altering working times or ensuring benefits to pregnant employees may also support women during their pregnancy; however, we need rigorous studies that directly examine the impact of policies on birth outcomes to truly understand the effect of such policies.

\section{CONCLUSION}

Our study uses a multidimensional definition of precarious employment. We found that women with higher employment precarity had higher risk of LBW. NonHispanic black and Hispanic women had elevated risk of LBW across levels of precarious employment. Our findings may inform workplace policies to better support women during pregnancy.

Acknowledgements We would like to thank Dr. Vanessa Oddo for her assistance and advice in downloading the NLSY data.

Contributors DMP was responsible for analysis and interpretation of the data as well as writing the paper. DAE substantively revised the manuscript for important intellectual content. TP contributed to the conception of the study and substantively revised the manuscript for important intellectual content. NS substantively revised the manuscript for important intellectual content. AH conceived and designed the study, conducted additional data analysis and contributed to interpretation of data. She also substantively revised the manuscript and oversaw the project. All authors reviewed and approved of the submitted version and are accountable for ensuring accuracy and integrity of the work. There is no one else who fulfils the criteria that has been excluded as an author.

Funding This work was supported in part by the Maternal and Child Health Bureau of the Health Resources and Services Administration (grant \#T76MC00011) and the National Institute of Aging (R01AG060011).

Competing interests None declared.

Patient consent for publication Not required.

Provenance and peer review Not commissioned; externally peer reviewed.

Data availability statement Data are publicly accessible through the US Bureau of Labor Statistic's website for National Longitudinal Surveys: https://www.nlsinfo.org/ content/cohorts/nlsy79/get-data.

Open access This is an open access article distributed in accordance with the Creative Commons Attribution Non Commercial (CC BY-NC 4.0) license, which permits others to distribute, remix, adapt, build upon this work non-commercially, and license their derivative works on different terms, provided the original work is properly cited, appropriate credit is given, any changes made indicated, and the use is non-commercial. See: http://creativecommons.org/licenses/by-nc/4.0/.

ORCID iD

Anjum Hajat http://orcid.org/0000-0001-8807-9232

\section{REFERENCES}

1 Wang S-F, Shu L, Sheng J, et al. Birth weight and risk of coronary heart disease in adults: a meta-analysis of prospective cohort studies. J Dev Orig Health Dis 2014;5:408-19. 
2 Zarrati M, Shidfar F, Razmpoosh E, et al. Does low birth weight predict hypertension and obesity in schoolchildren? Ann Nutr Metab 2013;63:69-76.

3 McCormick MC. The contribution of low birth weight to infant mortality and childhood morbidity. N Engl J Med 1985;312:82-90.

4 Hack M, Flannery DJ, Schluchter M, et al. Outcomes in young adulthood for very-low-birth-weight infants. $N$ Engl J Med 2002;346:149-57.

5 Behrman JR, Rosenzweig MR. Returns to birthweight. Review of Economics and Statistics 2004;86:586-601.

6 Martin JA, Hamilton BE, Osterman MJ, et al. Births: final data for 2016, 2018.

7 Valero De Bernabé J, Soriano T, Albaladejo R, et al. Risk factors for low birth weight: a review. Eur J Obstet Gynecol Reprod Biol 2004:116:3-15.

8 United States Department of Labor. Women's Bureau (WB) - Women in the Labor Force.

9 Mathews TJ, Hamilton BE. Mean age of mothers is on the rise: United States, 2000-2014. NCHS data brief 2016:1-8.

10 Burdorf A, Figa-Talamanca I, Jensen TK, et al. Effects of occupational exposure on the reproductive system: core evidence and practical implications. Occup Med 2006;56:516-20.

11 Figa-Talamanca I. Occupational risk factors and reproductive health of women. Occup Med 2006;56:521-31.

12 Henriksen TB, Hedegaard M, Secher NJ. Standing and walking at work and birthweight. Acta Obstet Gynecol Scand 1995;74:509-16.

13 Florack EIM, Pellegrino A, Zielhuis GA, et al. Influence of occupational physical activity on pregnancy duration and birthweight. Scand J Work Environ Health 1995;21:199-207.

14 Palmer KT, Bonzini M, Harris EC, et al. Work activities and risk of prematurity, low birth weight and pre-eclampsia: an updated review with meta-analysis. Occup Environ Med 2013;70:213-22.

15 Work KVL. And work-related stress in pregnancy. Clin Obstet Gynecol 2012:55:765-73.

16 Lee B-E, Ha M, Park H, et al. Psychosocial work stress during pregnancy and birthweight. Paediatr Perinat Epidemiol 2011;25:246-54.

17 Karasek RA, Demands J, Latitude JD. And mental strain: implications for job redesign. Adm Sci Q 1979;24:285-308.

18 Peckham TK, Baker MG, Camp JE, et al. Creating a future for occupational health. Annals of Work Exposures and Health 2017;61:3-15.

19 Weil D. The fissured workplace: Why work became so bad for so many and what can be done to improve it. Cambridge, Massachusetts London: Harvard University Press, 2014.

20 Bosch G. Towards a new standard employment relationship in Western Europe. Br J Ind Relat 2004:42:617-36.

21 Kalleberg AL. Good jobs, bad jobs: the rise of polarized and precarious employment systems in the United States, 1970s to 2000s. New York, NY: Russel Sage Foundation, 2013.

22 Benach J, Vives A, Amable M, et al. Precarious employment: understanding an emerging social determinant of health. Annu Rev Public Health 2014;35:229-53.

23 Scott-Marshall H, Tompa E. The health consequences of precarious employment experiences. Work 2011;38:369-82.

24 Dooley D, Prause J, Weight B. Birth weight and mothers' adverse employment change. J Health Soc Behav 2005;46:141-55.

25 Kalleberg AL, Work P. Precarious work, insecure workers: employment relations in transition. Am Sociol Rev 2009;74:1-22.

26 Julià M, Vanroelen C, Bosmans K, et al. Precarious employment and quality of employment in relation to health and well-being in Europe. Int J Health Serv 2017;47:389-409.

27 Eurofound. Quality of employment conditions and employment relations in Europe. Dublin, Ireland: Eurofound, 2013.

28 Lewchuk W, Clarke M, de Wolff A. Working without commitments: precarious employment and health. Work, Employment and Society 2008:22:387-406.
29 Sandman CA, Wadhwa PD, Dunkel-Schetter C, et al. Psychobiological influences of stress and HPA regulation on the human fetus and infant birth outcomes. Ann N Y Acad Sci 1994:739:198-210.

30 O'Donnell K, O'Connor TG, Glover V. Prenatal stress and neurodevelopment of the child: focus on the HPA axis and role of the placenta. Dev Neurosci 2009;31:285-92.

31 Sandman CA, Wadhwa PD, Chicz-DeMet A, et al. And Fetal/Infant Outcomea. Ann N Y Acad Sci 1997;814:266-75.

32 Tompa E, Scott-Marshall H, Dolinschi R, et al. Precarious employment experiences and their health consequences: towards a theoretical framework. Work 2007;28:209-24.

33 Lane SD, Keefe RH, Rubinstein R, et al. Structural violence, urban retail food markets, and low birth weight. Health Place 2008;14:415-23.

34 Luo Z-C, Wilkins R, Kramer MS. Effect of neighbourhood income and maternal education on birth outcomes: a population-based study. Can Med Assoc J 2006;174:1415-20.

35 Phillippi JC. Women's perceptions of access to prenatal care in the United States: a literature review. J Midwifery Womens Health 2009:54:219-25.

36 Casas M, Cordier S, Martínez D, et al. Maternal occupation during pregnancy, birth weight, and length of gestation: combined analysis of 13 European birth cohorts. Scand J Work Environ Health 2015;41:384-96

37 Larsen AD, Hannerz $\mathrm{H}$, Juhl $\mathrm{M}$, et al. Psychosocial job strain and risk of adverse birth outcomes: a study within the Danish national birth cohort. Occup Environ Med 2013;70:845-51.

38 Lu MC, Halfon N. Racial and ethnic disparities in birth outcomes: a life-course perspective. Matern Child Health J 2003;7:13-30.

39 Cramer JC. Racial and ethnic differences in birthweight: the role of income and financial assistance. Demography 1995;32:231-47.

40 Menéndez $\mathrm{M}$, Benach $\mathrm{J}$, Muntaner $\mathrm{C}$, et al. Is precarious employment more damaging to women's health than men's? Soc Sci Med 2007;64:776-81.

41 Hipple S. Contingent work in the Late-1990s. Mon Labor Rev 2001;124.

42 Kott PS. Clarifying some issues in the regression analysis of survey data. InSurvey research methods. , 2007: Vol. 1, 11-18No. 1.

43 Solon G, Haider SJ, Wooldridge JM. What are we weighting for? J Hum Resour 2015;50:301-16.

44 VanderWeele TJ, Knol MJ. A tutorial on interaction. Epidemiol Method 2014;3:33-72.

45 Larsen AD. The effect of maternal exposure to psychosocial job strain on pregnancy outcomes and child development. Dan Med J 2015;62:B5015.

46 Homer CJ, Beresford SAA, Jarnes SA, et al. Work-Related physical exertion and risk of preterm, low birthweight delivery. Paediatr Perinat Epidemiol 1990;4:161-74.

47 Vives A, Amable M, Ferrer M, et al. The employment precariousness scale (EPRES): psychometric properties of a new tool for epidemiological studies among waged and salaried workers. Occup Environ Med 2010;67:548-55.

48 Dong Y, Peng C-YJ. Principled missing data methods for researchers. Springerplus 2013;2:222.

49 Jurek AM, Greenland S, Maldonado G, et al. Proper interpretation of non-differential misclassification effects: expectations vs observations. Int J Epidemiol 2005;34:680-7.

50 Jurek AM, Greenland S, Maldonado G. How far from non-differential does exposure or disease misclassification have to be to bias measures of association away from the null? Int $J$ Epidemiol 2008;37:382-5.

51 Tomeo CA, Rich-Edwards JW, Michels KB, et al. Reproducibility and validity of maternal recall of pregnancy-related events. Epidemiology 1999:10:774-6. 